\title{
Checking the Temporal Behaviour of Distributed and Parallel Embedded Systems
}

\author{
Wolfgang A. Halang \\ Fernuniversität \\ Fachbereich Elektrotechnik und Informationstechnik \\ 58084 Hagen, Germany \\ wolfgang.halang@fernuni-hagen.de
}

\author{
Nihal Kececi \\ Université du Québec à Montréal \\ Département d'informatique \\ Montréal, Québec H3C 3P8, Canada \\ nkececi@lrgl.uqam.ca
}

Grace Tsai

Fairleigh Dickinson University

Department of Computer Science

Teaneck, NJ 07666, U.S.A.

tsai@alpha.fdu.edu

\begin{abstract}
An independent test facility is described, which simulates the environments of distributed and parallel embedded real time systems with special emphasis on the exact modeling of the prevailing time conditions. Its main application areas are software verification and safety licensing. Following the black box approach, just by providing worst case oriented input patterns to integrated hardware/software systems and monitoring the corresponding outputs, the time behaviour of such systems can precisely be determined. High accuracy time information is provided by employing a hardware supported timer synchronised with legal time, viz., Universal Time Co-ordinated, as received via GPS satellites.
\end{abstract}

Keywords: Black box testing, software verification, simulation, safety licensing, high precision timing, GPS. 


\section{INTRODUCTION}

The problems encountered in establishing proper real time behaviour of distributed or parallel embedded systems are difficult and manifold, and, hence, not satifactorily solved, yet. They are exacerbated by the need to prove that the timing behaviour of integrated hardware/software systems meets given specifications. This holds, in particular, for systems working in safety related environments, which must be approved by licensing authorities in a fully independent manner.

The main requirement real time embedded systems must fulfill is that their operation keeps pace with external processes, i.e., the acquisition and evaluation of process data, as well as appropriate reactions, must be carried through on time in synchrony with the events occurring in these embedding processes. It is a difficult design issue to guarantee punctuality of real time systems, because the response times depend on the computers' actual workload and the operating system overhead, and may differ from one external request to the next.

Only in trivial cases and when not using operating systems it may be possible to predict time behaviour by analysing program code. The common testing practice of instrumenting programs with output statements to obtain information on intermediate programs states is not applicable to timing analysis, because it is intrusive and, thus, alters the time behaviour. Furthermore, tests in actual application environments are often either too expensive, too dangerous, or simply impossible for other reasons. Hence, such environments must be simulated, and the black box approach has to be taken in examining embedded systems, i.e., they may not be modified under any circumstances, and any internal details are disregarded. It is only observed whether instants and values of outputs generated conform to the given requirements. Thus, maximum objectivity in quality assurance and safety licensing is guaranteed.

In this paper it is shown that an examined distributed or parallel embedded system's environment can effectively be simulated, and its operation in the time dimension be supervised and monitored using standard real time computers extended by a number of hardware and software features. The software implemented in the embedded system under examination is checked by one or more test plans prescribing external stimuli and their respective timing. These test plans are totally independent on the examined software, and are executed on different machines. Hence, this software verification method appears to be a candidate for recognition as an official safety licensing procedure. 


\section{FUNCTIONALITY OF THE TEST FACILITY}

In contrast to the conventional white box testing, which requires information about the testees' implementation, black box testing allows tests solely on the basis of specifications (cf. [1] for an introduction). Since

- the hardware and software for generating and evaluating test data is totally independent of testee implementation, thus allowing their development by other people - even at the same time as the implementation, and as

- the tests start at the external (standard) interfaces settled in the specifications suggesting the use of universal test environments,

the here pursued approach seems especially suitable for safety licensing.

Nevertheless, some of the "ingredients" necessary for this were applied for similar purposes already rather early. Thus, for a long time it is quite customary to use simulators for testing process control computer systems (leaving aside the classical testing of VLSI hardware being inconceivable without simulation) in some specialised industrial domains; for examples see $[8,3,4,6,5]$.

Furthermore, the use of monitoring systems to observe the behaviour of computer systems was advocated for in the scientific literature, especially for error detection in distributed systems (see [7] for an overview). Though, the interactivity of this search process makes it almost indispensable to provide mechanisms for re-runs (see, e.g., [14]). Especially in the domain of real time systems, even pure monitoring is feasible (see [9]), particularly to verify the observance of logical and, above all, timing conditions.

Though, there are only few publications (such as $[12,11])$ about integrated test environments with monitoring and simulation components. The mentioned contributions are tailored towards special architectures and mainly designed for white box testing. A good survey of this established 1980 in [2] and still existing - "lost world" of industrial software testing practices in the real time domain can be found in [13].

According to the application conditions mentioned in the introduction, a testing environment useful to support safety licensing of integrated hardware/software systems operating under real time conditions needs to provide the following services:

- simulation of the environment, in which an examined embedded system is working, by generating reproducible input patterns oriented at typical and worst case conditions, 
- surveillance if the outputs produced by the examined system are correct and appear within given time limits,

- no interference with the examined system, particularly no lengthening of its program code and execution time,

- interfacing to the examined system with the same hardware connectors as in the actual operating environment,

- access to the legal time, i.e., Universal Time Co-ordinated (UTC), for correct time stamping of events, correct timing of simulated external events, and to allow for putting distributedly acquired monitoring data into correct relation, which are obtained when examining distributed or parallel systems,

- free and easy programming (or configuration) of arbitrary test plans, and

- providing easily readable, concise reports on the test results.

\section{HARDWARE ARCHITECTURE OF THE TEST FACILITY}

A prototype of a simulation unit meeting the requirements compiled in the preceding section was built. For an overview on the hardware structure of the unit we refer to Figure 1.

Grouped along the internal I/O bus of a standard microprocessor, there is a video terminal to operate the unit, a printer for the reports, and a mass storage device. The latter may hold larger data sets to be provided as inputs to the embedded system examined, besides the files needed internally. In accordance with the system's interconnection pattern to its environment, the simulation unit is equipped with process peripherals such as digital interfaces, analogue converters, and IEC 488 attachments as well as with various serial line interfaces. Since the number and type of these connections varies with different systems examined, there is the possibility to insert corresponding peripherals into I/O slots of the unit. All external lines are brought to appropriate plugs, to enable easy connection to various systems.

In contrast to this, the further attachments mentioned now are always present. A bidirectional interface to the examined system's I/O data bus allows to simulate other peripheral devices. Their addresses are freely selectable. A number of registers is provided that work independently and in parallel, into which the microcomputer can store such addresses. Each register's output and the I/O address bus lines of the system on 


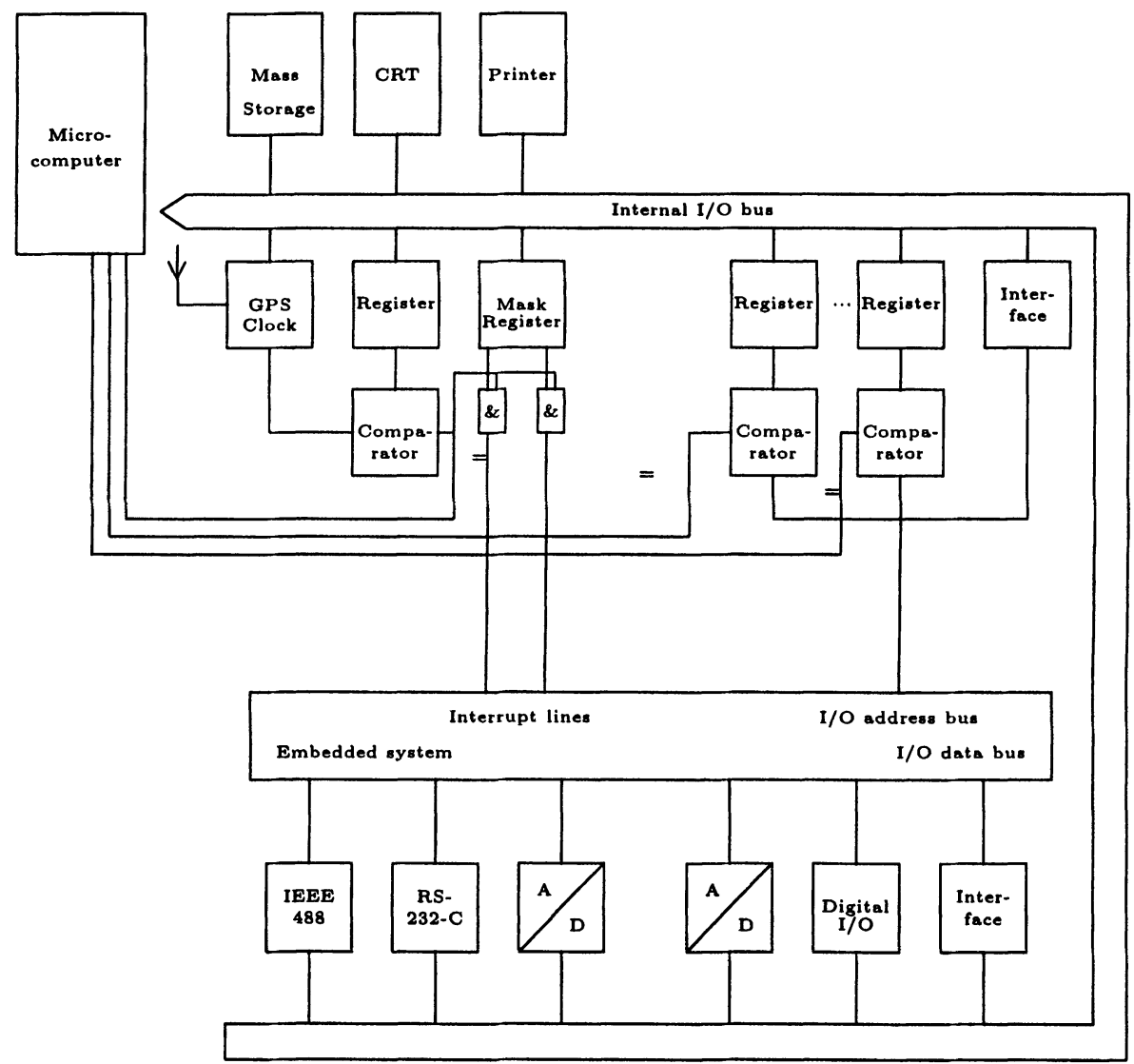

Figure 1. Hardware architecture of a testing facility (handling one node each of a distributed or parallel embedded system) 
test are fed into corresponding hardware comparators which send signals to the microprocessor when they detect matches of their inputs.

A similar feature is combined with the system clock in order to generate, as specified in test plans, exactly timed signals, to be supplied to the interrupt input lines of the examined system. The microprocessor always loads the next of these interrupt times into the comparison register. Upon equality with the clock, the comparator raises a signal that is transmitted to the microprocessor, where it initiates an associated routine, and to all interrupt lines whose corresponding bits in the mask register are set. This interrupt generation feature with exact timing is indispensable for a software verification device, because the environment of an examined system must be modeled as closely as possible, and because time signals delayed by randomly distributed durations may lead to erroneous test results. The feature is not only employed to feed interrupt signals into an examined system, but also to initiate data inputs synchronised with a time pattern.

\section{SOFTWARE COMPONENTS OF THE TEST FACILITY}

The environment simulator is furnished with a real time multitasking operating system, and a programming environment containing, as the main component, a high level process control language, extended by a few features that support the special hardware. In this language, test plans are written which formally specify the requirements embedded systems have to fulfill. For each interrupt source to be simulated, a test plan contains a time schedule that generates periodic or randomly distributed interrupts according to worst case conditions. The identifications and the occurrence times of these interrupts are recorded for later usage in performance reports. According to the temporal patterns of interrupts, the test plan processor writes appropriate data to the different outputs that are fed into an examined system. Analogously, the test plan specifies those events, coming from an examined system, that are to be awaited, and the reactions to be carried out upon their occurrence.

With the help of the I/O address comparison features the inputs of the environment simulator are supervised to determine a tested system's reaction times, and whether it provides correct output values. Together with their sources and their arrival times these data are also recorded to be used in the final reports. Since only the external reactions to a given workload are considered, the simulation method takes the operating system overhead into account, too. By the possible interconnection of the $\mathrm{I} / \mathrm{O}$ busses of the testing and the tested device, any kind of peripher- 
als, including DMA units, can be simulated. To carry this through, a test plan only needs to specify a suitable data source or sink, and an appropriate transfer rate. Further useful functions, that can be invoked in test plans, are $\mathrm{I} / \mathrm{O}$ bus traces and the logging of $\mathrm{I} / \mathrm{O}$ port activities with time specifications, to be provided in appropriate buffer areas of the simulation unit.

\section{TIMING OF HIGHEST ACCURACY}

An important aspect for the assessment of distributed and parallel real time embedded systems is the observability of the environment, i.e., it must be possible to observe every event of interest generated by the external (the embedding) process, and to determine their correct timing as well as temporal and causal order. This holds in particular for simultaneously occurring events in distributed systems whose simultaneity will, of course, only be recognised at a later stage when they will be (sequentially) processed. Also, this is a prerequisite for correctly processing avalanches of asynchronous interrupts. To enable observability and to establish information consistency between internal real time data bases and the environment, distributed and parallel real time embedded systems - and corresponding testing facilities - require a common time base to measure the absolute time of event occurrences.

For the correctly timed generation of outgoing stimuli and the time stamping of all monitored process events the environment simulator employs a high precision timer and interrupt controller as depicted in Figure 2, whose original design was described in [15]. It uses the correct time information broadcast via the Global Positioning System (GPS).

Alarm jobs, i.e., time instants coded in 32 bits with a resolution of $100 \mu \mathrm{sec}$ including seconds, minutes, and hour of the day, and unique alarm numbers identifying associated activities, are submitted to the timing controller using an 8 bits wide data bus. Submissions may arrive at any time. A novel Smallest Input First Out (SIFO) memory is used to keep a sorted list of alarm jobs. Its function is similar to the one of the well-known FIFO with the exception that it is not just a buffer but automatically sorts the incoming data, too. In this application, the SIFO also has to assemble the incoming 8 bits wide data stream to alarm job descriptors of 40 bits each. This assembling and the sorting need some time and, therefore, an additional input FIFO is used to ensure zero delay data input. The last SIFO location holds the alarm job descriptor with the next due date. If the timer receives an alarm job due even earlier, then this one will replace the former. However, the contents of the last SIFO location is always presented at the data output. If the 


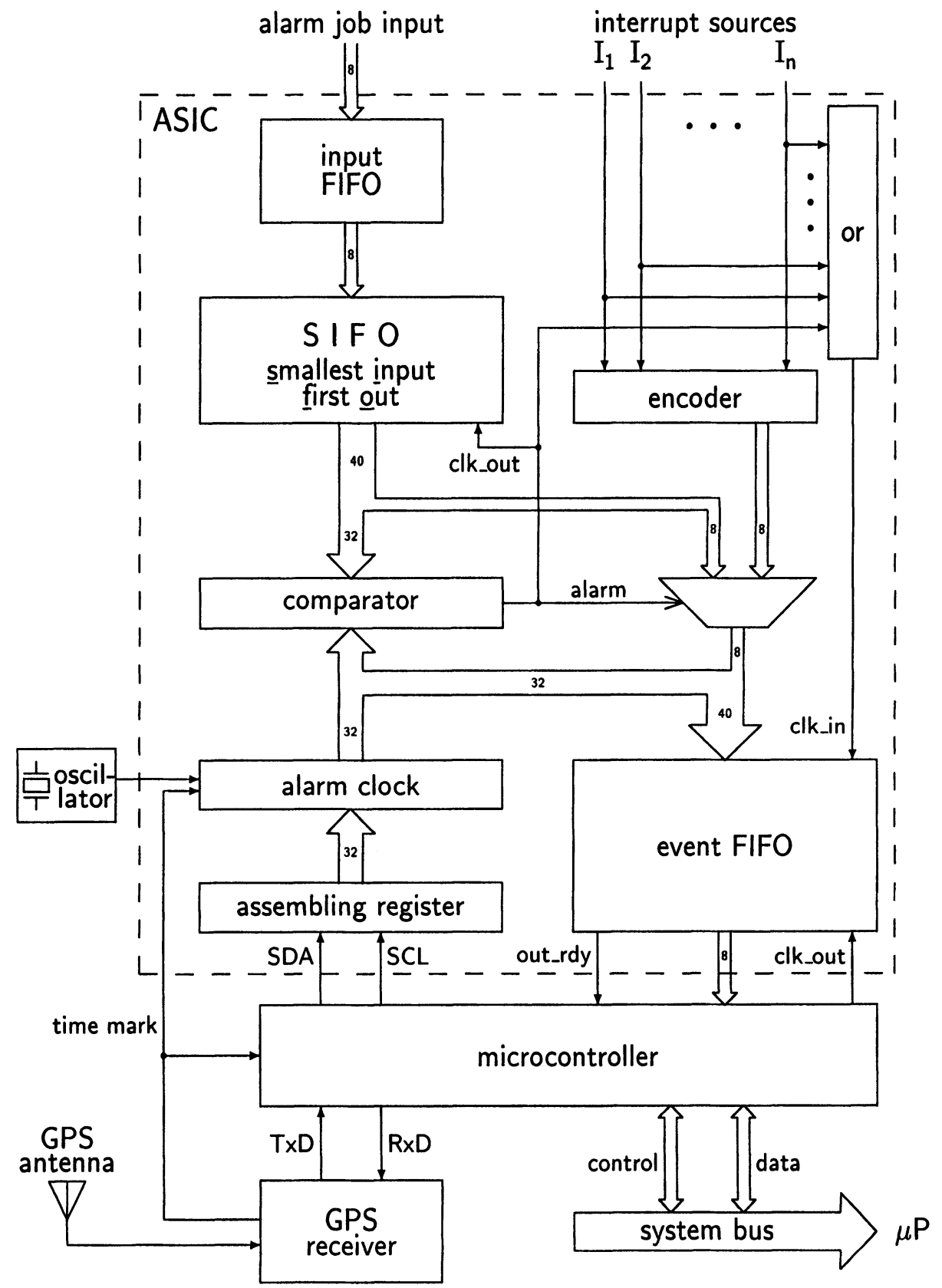

Figure 2. Functional diagram of a high precision timing and interrupt controller 
due time of the alarm job at the SIFO output is (smaller or) equal to the actual time as given by the alarm clock, then the comparator generates an alarm signal, which triggers the output (event) FIFO to read the actual time and the corresponding alarm number from the SIFO. To prevent delays, the SIFO immediately forwards the next alarm job descriptor to its output.

For time stamping all monitored process events, the high precision time processor was now extended by an interrupt receptacle. When an interrupt signal occurs at one of the newly provided input lines $I_{1} \ldots I_{n}$, a corresponding time stamp is formed by the interrupt's arrival time and the encoded interrupt line number. The time stamp descriptor is latched into the event FIFO. Until they will be read out by the microcontroller, the event FIFO buffers the alarm job and interrupt descriptors.

As shown in Figure 2, this high precision timer and interrupt controller consists of an application specific integrated circuit (ASIC) implementing an alarm job handler and a time stamping unit for arriving interrupts, a GPS receiver with attached antenna, and a microcontroller interfacing the GPS receiver to the ASIC. The information obtained by the GPS receiver includes, among others, UTC time and date, position and GPS status. It is transmitted via a serial data interface to the microcontroller, which sends commands to the receiver for configuration purposes and requests specific data. At system set-up and every midnight the time information is transferred into the alarm clock inside the ASIC using a synchronous serial link. To this end, first single bytes of information are assembled in a corresponding register and, then, transferred to the alarm clock. Thus, the alarm clock keeps track with leap seconds and the beginning of months. Our alarm clock prototype has a resolution of $100 \mu \mathrm{sec}$. It is driven by a free running oscillator and synchronised with UTC every second using the time mark signal as provided by the GPS receiver, which has an accuracy of $\pm 1 \mu$ sec. The microcontroller monitors the time mark signal. The controller is also used to read out the event FIFO as soon as it contains data. It keeps a list of all time and event driven activities sorted according to increasing deadlines.

\section{CONCLUSION AND OUTLOOK}

A very important subject of future reserach activities will be the automatical generation of test plans from specifications, i.e.,

- (automatical) generation of test plans and test data for all standardisable testing options, and

- (automatical) checking of the test results 
for non-trivial specifications, requiring to join formal and experimental verification. Naturally, the idea of creating appropriate test plans for any kind of testees and test options to be investigated with the help of expert systems is unavoidable. Such expert systems will be similar to those ones already used for the detection of hardware malfunctions.

\section{REFERENCES}

[1] R.A. DeMillo, W.M. McCracken, R.J. Martin and J.F. Passafiume: Software Testing and Evaluation. Benjamin Cummings Publ., 1987.

[2] R.L. Glass: Real-Time: The "Lost World" of Software Debugging and Testing. Comm. ACM, 23, 5, 264-271, 1980.

[3] G. Hauser: Test von Steuerungssoftware mit Hilfe der Realzeitsimulation. In F. Breitenecker and W. Kleinert (Eds.), Informatik-Fachberichte 85, pp. 495-499, Springer-Verlag, 1984.

[4] K. Hellmold: Multiprozessorsystem für die parallele Simulation von zeitdiskreten Systemen. In M. Goller (Ed.), Informatik-Fachberichte 56, pp. 227233, Springer-Verlag, 1982.

[5] H.B. Keller: Verteilte/modulare Echtzeitsimulation komplexer Systeme. In W. Ameling (Ed.), Informatik-Fachberichte 179, pp. 73-83, Springer-Verlag, 1988.

[6] R. Kodweiß: Software-Konzept für Echtzeit-Simulation. In D. Möller (Ed.), Informatik-Fachberichte 109, pp. 251-253, Springer-Verlag, 1985.

[7] C.E. McDowell and D.P. Helmbold: Debugging Concurrent Programs. ACM Computing Surveys, 21, 4, 593-622, 1989.

[8] J.M. Mohan and M. Geller: An Environmental Simulator for the FDNY Computer-Aided Dispatch System. In R.L. Glass (Ed.), Real-Time Software, pp. 75-90, Prentice-Hall, 1983.

[9] U. Schmid: Monitoring Distributed Real-Time Systems. Real-Time Systems, 7, 33-56, 1994.

[10] U. Schmid: Synchronized Universal Time Coordinated for Distributed RealTime Systems. IFAC Control Engineering Practice, 1995.

[11] E. Schoitsch, E. Dittrich, S. Grasegger, D. Kropfitsch, A. Erb, P. Fritz and H. Kopp: The ELEKTRA Testbed: Architecture of a Real-Time Test Environment for High Safety and Reliability Requirements. Proc. IFAC SAFECOMP '90, Gatwick, pp. 59-65, 1990.

[12] W. Schütz: A Test Strategy for the Distributed Real-Time System MARS. Proc. IEEE CompEuro'90, Tel Aviv, pp. 20-27, 1990.

[13] W. Schütz: The Testability of Distributed Real-Time Systems. Ph.D. Thesis, Technical University of Vienna, 1992.

[14] J.P. Tsai, K.-Y. Fang, H.-Y. Chen and Y.-D. Bi: A Noninterfering Monitoring and Replay Mechanism for Real-Time Software Testing and Debugging. IEEE Transactions on Software Engineering, SE-16, 8, 897-916, 1990.

[15] W.A. Halang, M. Wannemacher, P.A. Laplante and A.D. Stoyen: High-Precision Temporal Synchronization in Multimedia Systems. In B. Furht (Ed.), Handbook of Multimedia Computing, CRC Press, 1998. 